\title{
Gabor Filter-based Multiple Enrollment Fingerprint Recognition
}

\author{
Fred Kaggwa \\ Mbarara University of Science \\ and Technology \\ Mbarara, Uganda
}

\author{
John Ngubiri \\ Department of Computer \\ Science, Makerere University \\ Kampala, Uganda
}

\author{
Florence Tushabe \\ Uganda Technology and \\ Management University \\ Kampala, Uganda
}

\begin{abstract}
Minutiae-based matching techniques have been widely used in the implementation of multiple enrollment fingerprint recognition systems. However, these techniques suffer the difficulty of automatically extracting all minutiae points due to failure to detect the complete ridge structures of a fingerprint. With poor quality fingerprint images, detection of minutiae points as well as describing all the local ridge structures is difficult. It is also difficult to quickly match two fingerprints that have a difference in the number of unregistered minutiae. Non-minutiae based techniques such as Gabor filtering are rich in terms of distinguishing features and can be used as an alternative since they capture both the local and global details in a fingerprint. This paper presents a Gabor filter-based approach; the first of the kind to implement a verification multiple enrollment based fingerprint recognition system. The Gabor filter-based multiple enrollment fingerprint recognition method was compared with a spectral minutiae-based method using two fingerprint databases; FVC 2000-DB2-A and FVC 2006-DB2-A. Although the minutiaebased method outperformed the Gabor filter-based method, the results attained from the later are promising and can be a good basis for implementing Gabor filter-based techniques in designing multiple enrollment based fingerprint systems.
\end{abstract}

\section{General Terms}

Fingerprint Recognition, Pattern Recognition, Security, Algorithms, Biometrics.

\section{Keywords}

Multiple enrollment, Gabor Filter-based matching, Spectral Minutiae-based matching, Recognition performance, memory consumption, matching speed.

\section{INTRODUCTION}

The notion of multiple enrollment in fingerprint recognition systems has been an interesting research area for long; where researchers have proposed the use of multiple fingerprint samples to extend information of single enrolled fingerprint images, to ensure reliability of the fingerprint images and also to improve the recognition performance/accuracy of fingerprint recognition systems [29]. Researchers such as [24],[25],[26], [27],[28] and others in the related work section have mostly concentrated on minutiae-based matching methods while setting up multiple enrollment based fingerprint recognition systems. Other than minutiae-based matching methods, correlation based methods like [20],[6],[17],[18],[19] and pattern based methods such as [34], [35], [36] have been used for verification, indexing and identification in fingerprint recognition; but have rarely been implemented in multiple enrollment based fingerprint recognition systems. Jain et al [2] point out that minutiaebased approaches suffer the difficulty of automatically extracting all minutiae points due to failure to detect the complete ridge structures of a fingerprint. Based on the above scenario, matching becomes a difficult process for the case of two fingerprints having different numbers of uncaptured minutiae points. Furthermore, it is also difficult to describe all the local ridge structures as minutiae points, hence making matching a difficult process. A general overview of the minutiae-based methods is that, with poor quality fingerprint images, detection of minutiae points would be difficult hence affecting their resulting performance. Non-minutiae based techniques such as Gabor filtering are rich in terms of distinguishing features and can be used as an alternative since they capture both the local and global details in a fingerprint. This paper presents a Gabor filter-based approach; the first of the kind to implement a verification multiple enrollment based fingerprint recognition system.

The remaining part of this paper is organized as follows: Section 2 provides the related work, Section 3 provides the descriptions of the databases used and an overview of the matching methods used. Section 4 explains how the experiments were setup and the environment in which they were implemented. Section 5 , presents the results, provides a discussion and also points out the possible future work. Section 6 concludes the paper while the last two sections provide the acknowledgements and references respectively.

\section{RELATED WORK}

Multiple enrollment for fingerprint recognition is an old study area that has received a vast amount of research [42]. Minutiae based techniques such as [3],[4],[5],[7],[12] and [41] have been widely used in designing multiple enrollment fingerprint recognition systems. Although minutiae-based techniques have been widely used [1], they suffer the difficulty of automatically extracting all minutiae points due to failure to detect the complete ridge structures of a fingerprint. It is also difficult to quickly match two fingerprints that have a difference in the number of unregistered minutiae. Furthermore, it is also difficult to describe all the local ridge structures as minutiae points, hence making matching a difficult process [2]. Minutiae extraction also takes a lot of time [37]. Gabor filter-based techniques such as [8],[38],[9],[10], [11],[39],[44],[16],[13],[14],[21],[22],[15], have also attracted a lot of interest in designing fingerprint recognition systems. Gabor based fingerprint matching techniques are known to be rich in terms of distinguishing features and can be used as an alternative since they capture both the local and global details in a fingerprint. Their resultant representation is scale, translation and rotation invariant. They also produce short fixed length feature vectors, which makes them appropriate for indexing, faster fingerprint matching and storage on smaller devices [37]. On analysis of the literature, it was observed that the current research in using Gabor filterbased techniques has mainly focused on single enrollment rather than multiple enrollment for fingerprint recognition. It was also noted that there has been little or no focus on the 
running time/speed as well as memory consumption while using Gabor filter-based techniques. These gaps were a critical motivating factor in carrying out this research; to determine the possibility of implementing Gabor filter-based techniques in multiple enrollment based fingerprint recognition systems.

\section{DATABASE DESCRIPTIONS AND MATCHING METHODS USED}

\subsection{Database Descriptions}

Two public (internationally known) fingerprint databases namely; FVC2000-DB2 [31] and FVC2006-DB2 [32], were used.

\subsubsection{The FVC2000-DB2 Database}

This database comprises fingerprint image samples taken from 110 people with 8 impressions per person generating a total of 880 fingerprints. These multiple samples were collected from untrained people, there were no attempts made to guarantee the least possible acquisition quality and the collection was done in two different sessions. However, for all experiments in this paper, set A (FVC2000-DB2-A of 100 individuals) of the whole database which contains a total of 800 fingerprints was used.

\subsubsection{The FVC2006-DB2 Database}

This database comprises fingerprint image sample taken from 150 people with 12 impressions per person generating a total of 1800 fingerprints. During the collection of fingerprints, there was no deliberate introduction of difficulties such as exaggerated distortion, large amounts of rotation and displacement, wet/dry impressions, etc. (as it was done in the previous editions), but the population in this database is more heterogeneous and also includes manual workers and elderly people. However, the final datasets were selected from a larger database by choosing the most difficult fingers according to a quality index, to make the benchmark sufficiently difficult for a technology evaluation. For all experiments in this paper, subset A (FVC2006-DB2-A of 140 individuals) with a total of 1680 fingerprints images was used.

\subsection{The Matching Methods Used}

This section presents a description of the matching methods used throughout all the experiments.

\subsubsection{Spectral Minutiae-based matching}

In this method [30], all the minutiae template sets from the fingerprint image sample are first extracted and then stored with unique identification (ID) names. The extracted minutiae sets are then transformed into a spectral minutiae form (referred to as Minutiae Spectrum) by representing them as a fixed-length feature vector which is invariant to translation. Within the minutiae spectrum form, rotation and scaling also become translations which can easily be compensated for. Once the transformation into a Spectral Minutiae representation is done, direct matching follows by correlation between the two Spectral images and a similarity score is generated.

\subsubsection{Gabor Filter-based matching}

In this method, the Gabor features of all input fingerprint image samples are first extracted like in [40]. Column vectors consisting of the Gabor features of the input fingerprint image samples are created. These feature vectors are normalized to zero mean and unit variance (to remove any noise originating from sensors as well as the grey level background which maybe generated because of the finger pressure differences), and then stored with unique identification (ID) names. Direct matching follows by calculating the Euclidean distance (see Equation 1-Eq1) between the two feature vectors; Fvecl and Fvec2 respectively originating from the two fingerprint samples to be compared. Based on this Euclidean distance $(E d)$ value attained, a matching score is computed such that; the higher the Euclidean distance $(E d)$, the lower the matching score and vice versa. The score is computed and standardized as shown in Equation 2 (Eq2) [23].

Euclidean Distance $(E d)=\operatorname{sum}\left((x-y) .{ }^{\wedge} 2\right) .{ }^{\wedge} 0.5 E q(1)$

Where $\mathrm{x}$ and $\mathrm{y}$ are the feature vectors; Fvec1 and Fvec2 respectively originating from the two fingerprint samples to be compared.

Matching score $=\frac{1}{(1+E d)}$

Where $E d$ is the Euclidean distance between the two feature vectors; Fvecl and Fvec 2 respectively originating from the two fingerprint samples to be compared

\section{EXPERIMENTAL SETUP AND IMPLEMENTATION ENVIRONMENT}

In this section, the setup of both the minutiae-based and Gabor filter-based multiple enrollment experiments is described as well as the computational environment in which they were implemented.

\subsection{The Multiple Enrollment Experimental setup}

Based on the database, different comparisons were performed during the experimental setup. In the FVC2000-DB2-A database which comprises 100 fingers with 8 samples per finger, each comparison was performed based on five fingerprints that were selected from the dataset. In this case, four of the five fingerprints were used as the reference fingerprints and one as the test fingerprint. Score level fusion based on Max Rule in [33] then followed by taking the maximum score amongst the four attained values. In the FVC2006-DB2-A database which comprises 140 fingers with 12 samples per finger, each comparison was performed based on seven fingerprints that were selected from the dataset. For this case, six of the seven fingerprints were used as the reference fingerprints and one as the test fingerprint. Again, Score level fusion based on the Max Rule in [33] then followed by taking the maximum score amongst the six attained values. Below we provide a description of the genuine and impostor pairs used for the multiple enrollment experiments.

\section{Genuine Pairs}

For multiple genuine pair matching in the FVC2000-DB2-A database, four permutation sets (shown in Table 4.1), Set1, Set2, Set3 and Set4 were established for multi-sample enrollment and single-sample verification. Based on the 8 samples per person, four fingerprints of the same person, each as a reference were chosen matching each of them with the fifth sample of that person as the test fingerprint. On the other hand, for multiple genuine pair matching in FVC2006-DB2-A database, six permutation sets (shown in Table 4.2), Set1, Set2, Set3, Set4, Set5 and Set6 were established for multisample enrollment and single-sample genuine verification. Based on the 12 samples per person, six fingerprints of the same person each as a reference were chosen, matching each of them with the seventh sample of that person as the test 
fingerprint. There was no particular procedure followed in creating the permutation sets. All the permutation sets were randomly formulated.

\section{Impostor Pairs}

For multiple impostor pair matching in FVC2000-DB2-A database, the first sample of an identity in the database was chosen and matched with the four multiple enrollment samples of the different identities. While for multiple impostor pair matching in FVC2006-DB2-A database, the first sample of an identity in the database was chosen and matched with the six multiple enrollment samples of the different identities.

Table 4.1: FVC2000-DB2-A database permutation sets of the impressions used for multi-sample enrollment and single-sample verification

\begin{tabular}{|c|c|c|}
\hline Permutation Sets & $\begin{array}{c}\text { Enrollment } \\
\text { Samples }\end{array}$ & $\begin{array}{c}\text { Verification } \\
\text { Samples }\end{array}$ \\
\hline Set1 & $1,2,3,4$ & $5,6,7,8$ \\
\hline Set2 & $1,3,5,7$ & $2,4,6,8$ \\
\hline Set3 & $1,2,7,8$ & $3,4,5,6$ \\
\hline Set4 & $1,5,6,7$ & $2,3,4,8$ \\
\hline
\end{tabular}

Table 4.2: FVC2006-DB2-A database permutation sets of the impressions used for multi-sample enrollment and single-sample verification.

\begin{tabular}{|c|c|c|}
\hline Permutation Sets & $\begin{array}{c}\text { Enrollment } \\
\text { Samples }\end{array}$ & $\begin{array}{c}\text { Verification } \\
\text { Samples }\end{array}$ \\
\hline Set1 & $1,2,3,4,5,6$ & $7,8,9,10,11,12$ \\
\hline Set2 & $1,3,5,7,9,11$ & $2,4,6,8,10,12$ \\
\hline Set3 & $1,2,3,10,11,12$ & $4,5,6,7,8,9$ \\
\hline Set4 & $1,7,8,9,10,11$ & $2,3,4,5,6,12$ \\
\hline Set5 & $1,3,5,8,10,12$ & $2,4,6,7,9,11$ \\
\hline Set6 & $1,6,7,8,9,10$ & $2,3,4,5,11,12$ \\
\hline
\end{tabular}

\subsection{The Implementation Environment}

All the experimentations and algorithms in this research were implemented in MATLAB 7.12.0 (R2011a). All experiments were carried out using an Intel(R) Core(TM) i5-3230M CPU $2.60 \mathrm{GHz}$, with $4 \mathrm{~GB}$ of RAM running a 64-bit Windows 8 Pro operating system. For the minutiae-based method, the VeriFinger 6.0.0.7 extractor was used to extract all the minutiae templates from all the fingerprint images in all the two databases. For the Gabor Filter-based method, the Gabor filter extractor in [40] was used. The MATLAB Elapsed Time (etime) function was used to calculate how long the algorithms took to complete a task from the start to the end. On the other hand, the MATLAB Profiler feature was used to monitor the peak memory consumption/usage for each algorithm (Minutiae-based and Gabor filter-based) during all the computations/experimentations.

\section{RESULTS, DISCUSSIONS AND FUTURE WORK}

This section presents the results, their discussion and the anticipated future work.

\subsection{Experiments on the FVC2000-DB2-A Fingerprint Database}

For this database, four permutation sets (Set1, Set2, Set3, and Set4) were formulated for multi-sample enrollment and single-sample genuine verification as well impostor verification. For each permutation set in both the minutiaebased method and the Gabor Filter-Based method, a multisample enrollment and single-sample verification was performed to check the recognition performance amongst the sets. In each set 400 genuine comparisons and 9900 impostor comparisons were generated. For the whole multiple enrollment experiments in both the minutiae-based method and the Gabor Filter-Based method, $100 \times 4 \times 4=1600$ genuine comparisons and $100 \times 99 \times 4=39600$ impostor comparisons were generated. The attained Equal Error Rates (EERs), matching speeds, and peak memory consumptions per set for both the minutiae-based method and the Gabor Filter-Based method are shown in Table 5.1.

\subsection{Experiments on the FVC2006-DB2-A Fingerprint Database}

For this database, six permutation sets (Set1, Set2, Set3, Set4, Set5 and Set6) were formulated for multi-sample enrollment and single-sample genuine verification as well impostor verification. For each permutation set in both the minutiaebased method and the Gabor Filter-Based method, a multisample enrollment and single-sample verification was performed to check the recognition performance amongst the sets. In each set 840 genuine comparisons and 19460 impostor comparisons were generated. For the whole multiple enrollment experiments in in both the minutiae-based method and the Gabor Filter-Based method, $140 \times 6 \times 6=5040$ genuine comparisons and 140 × 139 × $6=116760$ impostor comparisons were generated. The attained Equal Error Rates (EERs), matching speeds, and peak memory consumptions per set for both the minutiae-based method and the Gabor Filter-Based method are shown in Table 5.2.

\subsection{Graphical Comparisons of Results from the FVC2000-DB2-A and FVC2006-DB2-A Fingerprint Database Experiments}

This section provides graphical comparisons resulting from the experiments done on both fingerprint databases. Figure 1(a) and Figure 1(b) provide comparisons on recognition performance, Figure 2(a) and Figure 2(b); running time/speed comparisons, while Figure 3(a) and Figure 3(b) provide comparisons on memory consumption. 


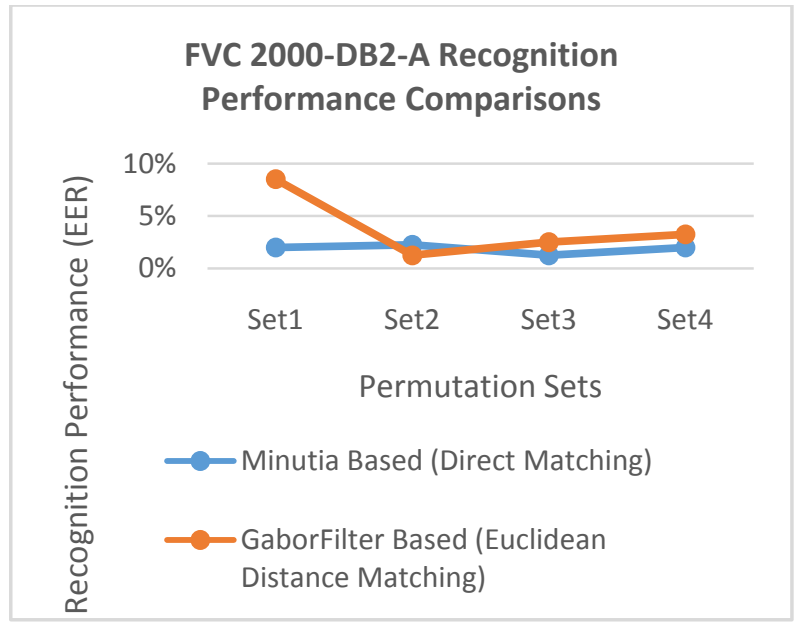

Figure 1(a): FVC 2000-DB2-A Recognition Performance Comparisons

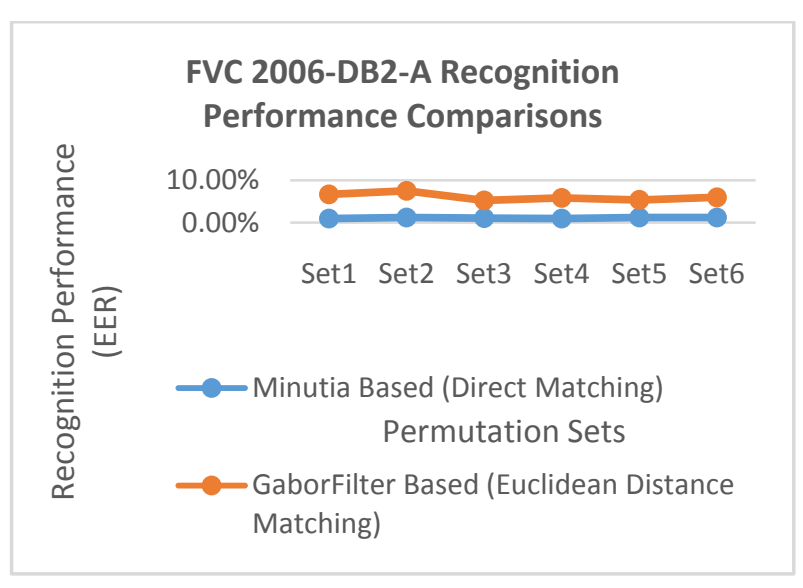

Figure 2(b): FVC 2006-DB2-A Recognition Performance Comparisons

Table 5.1: Summary of Experimentation Results on FVC 2000 DB2-A Database

\begin{tabular}{|c|c|c|c|c|c|c|}
\hline $\begin{array}{c}\text { FVC 2000- } \\
\text { DB2-A }\end{array}$ & \multicolumn{2}{|c|}{$\begin{array}{c}\text { Recognition } \\
\text { Performance (EER) }\end{array}$} & \multicolumn{2}{c|}{$\begin{array}{c}\text { Running Time } \\
\text { /Speed (sec) }\end{array}$} & \multicolumn{2}{c|}{$\begin{array}{c}\text { Peak Memory } \\
\text { Consumption(KB) }\end{array}$} \\
\hline $\begin{array}{c}\text { Permutation } \\
\text { Sets }\end{array}$ & $\begin{array}{c}\text { Minutiae- } \\
\text { Based }\end{array}$ & $\begin{array}{c}\text { Gabor Filter- } \\
\text { Based }\end{array}$ & $\begin{array}{c}\text { Minutiae- } \\
\text { Based }\end{array}$ & $\begin{array}{c}\text { Gabor Filter- } \\
\text { Based }\end{array}$ & $\begin{array}{c}\text { Minutiae- } \\
\text { Based }\end{array}$ & $\begin{array}{c}\text { Gabor Filter- } \\
\text { Based }\end{array}$ \\
\hline Set1 & $2 \%$ & $8.5 \%$ & 337.01 & 1609.45 & 960 & 2696 \\
\hline Set2 & $2.25 \%$ & $1.25 \%$ & 235.38 & 1561.27 & 320 & 1892 \\
\hline Set3 & $1.25 \%$ & $2.5 \%$ & 231.75 & 1588.27 & 148 & 1892 \\
\hline Set 4 & $2 \%$ & $3.25 \%$ & 232.82 & 1580.76 & 148 & 2084 \\
\hline
\end{tabular}

Table 5.2: Summary of Experimentation Results on FVC 2000 DB2-A Database

\begin{tabular}{|c|c|c|c|c|c|c|}
\hline $\begin{array}{c}\text { FVC 2006- } \\
\text { DB2-A }\end{array}$ & \multicolumn{2}{|c|}{$\begin{array}{c}\text { Recognition } \\
\text { Performance (EER) }\end{array}$} & \multicolumn{2}{c|}{$\begin{array}{c}\text { Running Time } \\
\text { /Speed (sec) }\end{array}$} & \multicolumn{2}{c|}{$\begin{array}{c}\text { Peak Memory } \\
\text { Consumption(KB) }\end{array}$} \\
\hline $\begin{array}{c}\text { Permutation } \\
\text { Sets }\end{array}$ & $\begin{array}{c}\text { Minutiae- } \\
\text { Based }\end{array}$ & $\begin{array}{c}\text { Gabor Filter- } \\
\text { Based }\end{array}$ & $\begin{array}{c}\text { Minutiae- } \\
\text { Based }\end{array}$ & $\begin{array}{c}\text { Gabor Filter- } \\
\text { Based }\end{array}$ & $\begin{array}{c}\text { Minutiae- } \\
\text { Based }\end{array}$ & $\begin{array}{c}\text { Gabor Filter- } \\
\text { Based }\end{array}$ \\
\hline Set1 & $0.95 \%$ & $6.67 \%$ & 594.58 & 13889.48 & 364 & 5536 \\
\hline Set2 & $1.19 \%$ & $7.50 \%$ & 593.4 & 13767.57 & 324 & 5404 \\
\hline Set3 & $1.07 \%$ & $5.24 \%$ & 723.78 & 10336.05 & 320 & 5024 \\
\hline Set4 & $0.95 \%$ & $5.83 \%$ & 704.87 & 10110.41 & 320 & 4828 \\
\hline Set5 & $1.19 \%$ & $5.36 \%$ & 622.81 & 10734.52 & 448 & 5216 \\
\hline Set6 & $1.19 \%$ & $5.95 \%$ & 547.95 & 10636.45 & 320 & 4896 \\
\hline
\end{tabular}




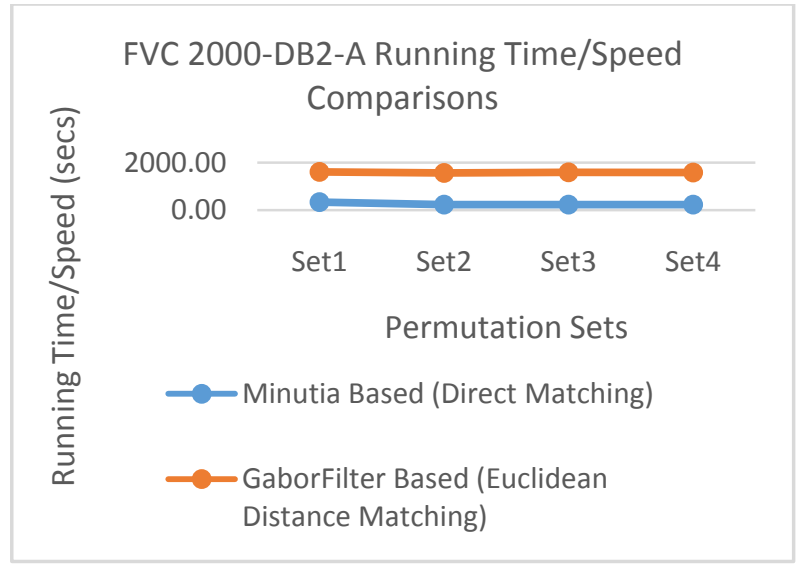

Figure 2 (a): FVC 2000-DB2-A Running Time/Speed Comparisons

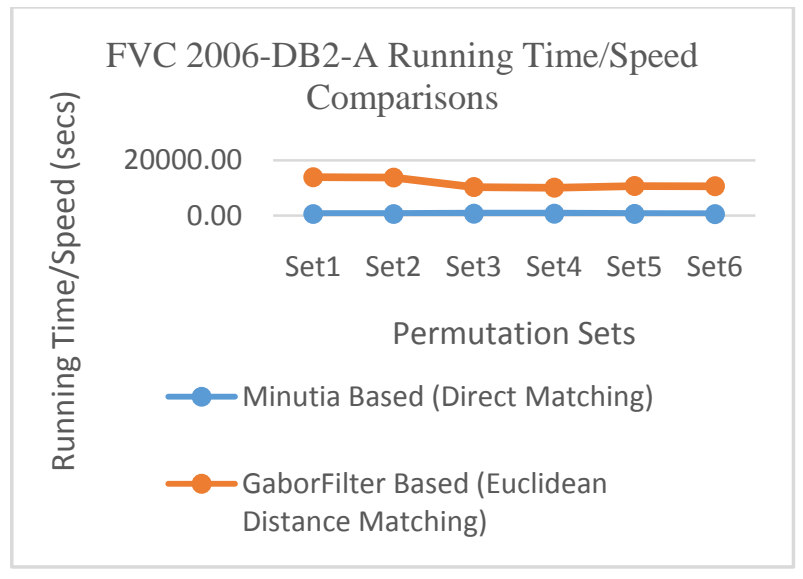

Figure 2 (b): FVC 2006-DB2-A Running Time/Speed Comparisons

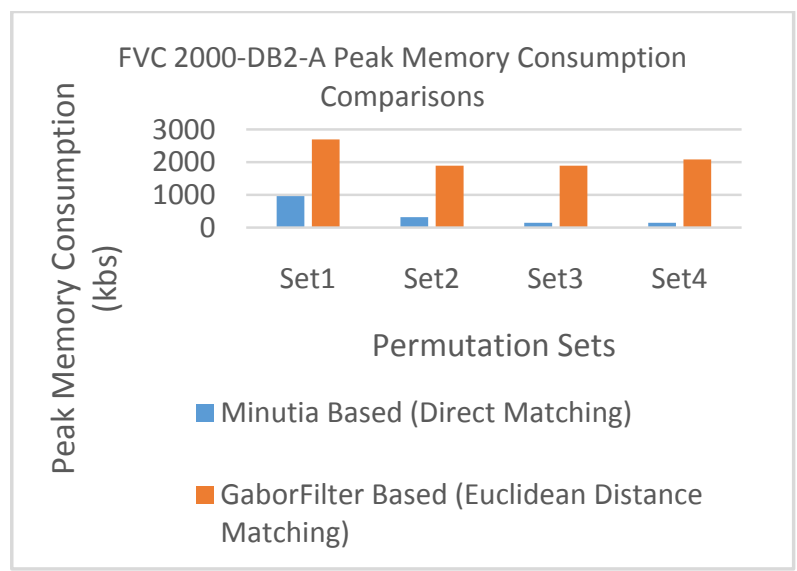

Figure 3(a): FVC 2000-DB2-A Peak Memory Consumption Comparisons

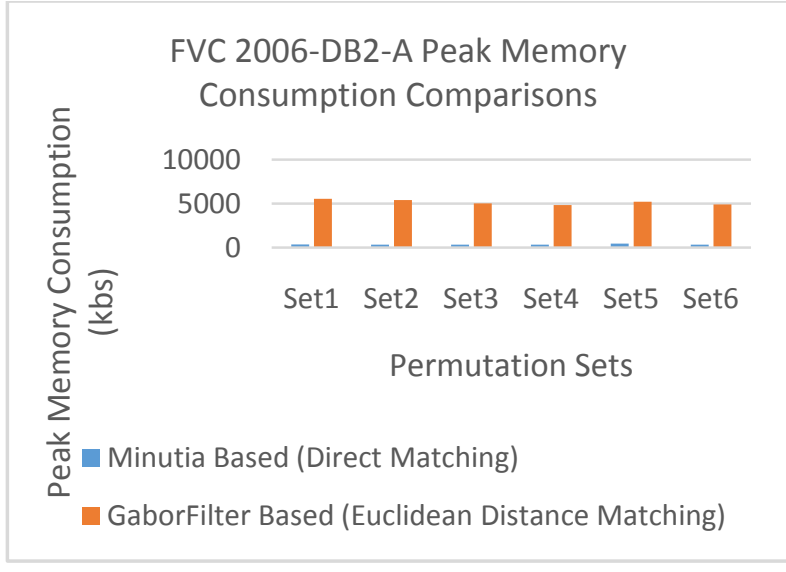

Figure 3(b): FVC 2000-DB2-A Peak Memory Consumption Comparisons

\subsection{Discussions and Future Work}

Critically analyzing the results from both fingerprint databases as presented in Table 5.1 and Table 5.2, one can clearly observe that minutiae-based approaches are still superior in terms of generating a good recognition performance, a reduced matching speed and a reduced memory consumption when implemented in multiple enrollment fingerprint recognition systems. However, based on the same results, one can anticipate that Gabor filter-based methods have a promising future for implementation in multiple enrollment based fingerprint recognition systems. For example, considering the results in Table 5.1, it can be observed that the recognition performance attained from experiments on Set2 in the FVC 2000-DB2-A fingerprint database under the Gabor filter-based method is far superior to that of the minutiae-based method.

A graphical illustration of the results as shown in Figures 1(a)\&1(b) for recognition performance, 2(a)\&2(b) for running time/speed and 3(a)\&3(b) for memory consumption; shows that the Gabor filter-based method has generally performed poorly with regards to recognition accuracy, running time/speed and memory consumption while the minutiae-based method performed better in all aspects. Based on the initial challenge identified in the minutiae based method as being difficult to extract all minutiae points from the fingerprint images, this already gives the minutiae method a less computation time/speed as well as a lower memory consumption; since the features to match are few. From our experiments, we observed that templates under the minutiaebased method had few features extracted. On the other hand, the feature vectors generated from the Gabor filter-based method were so rich with many features extracted. One can therefore confidently conclude that the many features contributed to the poor matching speed/running time as well as a higher memory consumption in the Gabor filter-based method.

It is however important to also note that in both methods the results are still not good enough as would be expected. This is attributed to the fact that there was no unique/additional advancement or tweaking performed onto the algorithms. In both cases (minutiae-based and Gabor filter-based), the algorithms match all the enrolled samples (i.e. whether good or bad) of an individual. This already puts an overhead to the matching speed as well as memory consumption; considering the fact that the more the images to match, the more time it takes and the more memory it consumes. 
Future work can consider unique advancements/adjustments to the algorithms of the Gabor filter-based method to cater for more acceptable results with regards to recognition performance, running time as well as memory consumption. Based on the suggestions the led to improved results in our previous research [43] towards improving recognition performance, speed and reducing memory consumption in minutiae based method, researchers could find alternative ways of identifying the samples of fingerprints amongst the many collected per individual that would yield good feature vectors and only match those when using the Gabor filterbased method. Future work can also deploy more techniques such as classification; for example k-Nearest Neighbor (KNN) classifier or the Support Vector Machines (SVM) classifier to improve on the matching of the feature vectors to attain a better recognition performance, matching speed and reduce memory consumption. In all our series, we have not handled the security of multiple templates. A vast amount of research has been done to secure single templates in single enrollment fingerprint recognition systems but little or no research has been dedicated to securing multiple templates in multiple enrollment fingerprint recognition systems. This could also be an important area to venture. Other interesting future research could be in implementing multiple enrollment in other biometrics such other face recognition where instead of capturing one copy of an individual's face, many copies would be captured with different poses.

\section{CONCLUSION}

This research aimed at implementing a non-minutiae based multiple enrollment fingerprint recognition method to observe recognition performance, running time/speed and memory consumption. The challenges of minutiae-based techniques have been discussed and an alternative suggested. As a proposition, a Gabor filter-based multiple enrollment fingerprint recognition method which was compared with a minutiae-based method for evaluations has been presented. Two fingerprint databases FVC 2000-DB2-A and FVC 2006DB2-A were used. Although the minutiae-based method outperformed the Gabor filter-based method, the results attained from the later are promising. The feature vectors in the Gabor filter-based method are so rich with enough distinguishing information compared to the minutiae templates. It is therefore realistic to mention that, with more tweaking and developments, the Gabor filter-based techniques can be a good choice for designing multiple enrollment based fingerprint recognition systems. Future work could focus on finding the samples of fingerprints amongst the many collected per individual that would yield good feature vectors and only match those when using the Gabor filter-based method to attain better results. Deployment of other techniques like classification using the k-Nearest Neighbor $(\mathrm{KNN})$ classifier or the Support Vector Machines (SVM) classifier would be paramount in improving the matching of the feature vectors to attain a better recognition performance, matching speed and reduce memory consumption.

\section{ACKNOWLEDGMENTS}

Great thanks to the experts; Assoc. Prof. John Ngubiri, Assoc. Prof. Florence Tushabe and Mr. Mohammad Haghighat who have contributed towards the success of this research.

\section{REFERENCES}

[1] Amira Saleh, Ayman Bahaa and A. Wahdan,"fingerprint Recognition," Computer and systems engineering department Faculty of Engineering /Ain Shams University, Egypt, 2011.
[2] Anil K. Jain, Fellow, IEEE, Salil Prabhakar, Lin Hong, and Sharath Pankanti, "Filterbank-Based Fingerprint Matching," IEEE Transactions On Image Processing, vol. 9, No. 5, 2000.

[3] Ishpreet Singh Virk and Raman Maini,“Fingerprint Image Enhancement and Minutiae Matching in Fingerprint Verification", Journal of Computing Technologies, vol. 1, Jun. 2012.

[4] Urvashi Chaudhary and Shruti Bhardwaj, "Fingerprint image enhancement and minutia extraction," International Journal of Advance Research in Computer Science and Management Studies, vol. 2, Issue 5, 2014.

[5] Raymond Thai, "Fingerprint Image Enhancement and Minutiae Extraction, the School of Computer Science and Software Engineering, the University of Western Australia, 2003.

[6] Prateek Verma, Maheedhar Dubey, Praveen Verma, "Correlation based method for identification of fingerprint- a biometricapproach," International Journal of Engineering and Advanced Technology (IJEAT), ISSN: 2249 - 8958, vol. 1, Issue 4, 2012.

[7] Manvjeet Kaur, Mukhwinder Singh, Akshay Girdhar, and Parvinder S. Sandhu, "Fingerprint Verification System using Minutiae Extraction Technique", World Academy of Science, Engineering and Technology 46 2008.

[8] Munir, M. U., Javed, M. Y., "Fingerprint Matching using Gabor Filters," 2005.

[9] E. Zhu, J. Yin, G. Zhang and C. Hu, "A Gabor filter based fingerprint enhancement scheme using average frequency", Int. Journal of Pattern Recog. and Artif. Intell., vol. 20, no. 3, pp. 417-429, 2006.

[10] C. J. Lee and S. D. Wang, "A Gabor filter-based approach to fingerprint recognition", Proc. IEEE Workshop on Signal Processing Systems (SiPS), pp. 371-378, 1999.

[11] F. Alonso-Fernandez, J. Fierrez-Aguilar and J. OrtegaGarcia, "An enhanced Gabor filter-based segmentation algorithm for fingerprint recognition systems", Proc. 4th Int. Symposium on Image and Signal Processing and Analysis (ISPA2), Zagreb, Croatia, pp. 239-244, 2005.

[12] A. K. Jain, A. Ross, and S. Prabhakar, "Fingerprint Matching Using Minutiae and Texture Features", Proc International Conference on Image Processing (ICIP), pp. 282-285, Greece, October 7-10, 2001.

[13] S. Prabhakar, "Gabor filter bank based Fingerprint classification and identification.", $\mathrm{PhD}$. Thesis, Department of Computer Engg., MSU

[14] Dhruv Batra, Girish Singhal and Santanu Chaudhury: Gabor Filter based Fingerprint Classification using Support Vector Machines, IEEE INDIA ANNUAL CONFERENCE 2004, INDICON 2004

[15] B. Garg, A. Chaudhary, K. Mendiratta and V. Kumar, "Fingerprint recognition using Gabor Filter," Computing for Sustainable Global Development (INDIACom), 2014 International Conference on, New Delhi, 2014, pp. 953958.

[16] M. U. Munir, M. Y. Javed, "Fingerprint Matching Using Gabor Filters In National Conference on Emerging Technologies (2004), pp. 147-151" 
[17] Bazen, Asker M., et al. "A Correlation-Based Fingerprint Verification System." (2000): 205-213.

[18] Nandakumar, Karthik, and Anil K. Jain. "Local correlation-based fingerprint matching." In Indian Conference on Computer Vision, Graphics and Image Processing. 2004.

[19] Krithika Venkataramani and B. V. K. Vijaya Kumar. 2003. Fingerprint verification using correlation filters. In Proceedings of the 4th international conference on Audio- and video-based biometric person authentication (AVBPA'03), Josef Kittler and Mark S. Nixon (Eds.). Springer-Verlag, Berlin, Heidelberg, 886-894.

[20] Almudena Lindoso, Luis Entrena, Judith Liu-Jimenez, and Enrique San Millan. 2007. Correlation-based fingerprint matching with orientation field alignment. In Proceedings of the 2007 international conference on Advances in Biometrics (ICB'07), Seong-Whan Lee and Stan Z. Li (Eds.). Springer-Verlag, Berlin, Heidelberg, 713-721.

[21] Alonso-Fernandez, Fernando, J. Fierrez, and Javier Ortega-Garcia. "An enhanced gabor filter-based segmentation algorithm for fingerprint recognition systems." Proc. IEEE Intl. Symposium on Image and Signal Processing and Analysis, ISPA, Spec. Sess on. Signal Image Processing for Biometrics, IEEE Press, Zagreb (Croatia), September 2005. University of Zagreb, 2005.

[22] Elmir, Youssef, et al. "Personal Identification by Fingerprints based on Gabor Filters." CIIA. 2009.

[23] Segaran, Toby. Programming Collective Intelligence: Building Smart Web 2. 0 Applications. Danbury: O'Reilly Media, Incorporated, 2007. Print.

[24] Mane, Arjun V., Yogesh S. Rode, and K. V. Kale. "Novel Multiple Impression based Multimodal Fingerprint Recognition System." International Journal of Computer Applications 27 (2011).

[25] Simon-Zorita D., Ortega-Garcia J., Sanchez-Asenjo M. and Gonzalez-Rodriguez J., "Facing Position Variability in Minutiae-Based Fingerprint Verification Through Multiple References and Score Normalization Techniques," in Proc. Int. Conf. on Audio- and VideoBased Biometric Person authentication (4th), pp. 214223, 2003a.

[26] C. Ren, Y. Yin, J. Ma, and G. Yang, "A Novel Method of Score Level Fusion Using Multiple Impressions for Fingerprint Verification. SMC," IEEE, pp. 5051-5056, 2009

[27] A.K. Jain, A. Ross and S. Prabhakar, "A hybrid fingerprint matching using minutiae and texture features", Proceedings of the International Conference on Image Processing (ICIP 2001), pp. 282-285

[28] C. Yang and J. Zhou. "A comparative study of combining multiple enrolled samples for fingerprint verification". Pattern Recognition, vol.39, no. 11, pp. 2115-2130, 2006.

[29] D. Maltoni, D. Maio, A.K. Jain, and S. Prabhakar, "Handbook of Fingerprint Recognition," Springer professional computing, Springer, 2009.

[30] Haiyun Xu, Raymond N.J. Veldhuis, Tom A.M. Kevenaar, Anton H.M. Akkermans, and Asker M. Bazen.
Spectral minutiae: A fixed-length representation of a minutiae set. Computer Vision and Pattern Recognition Workshop, 0:16, 2008

[31] D. Maio, D. Maltoni, J. L.Wayman, and A. K. Jain, "Fvc2000: Fingerprint verification competition," IEEE Transactions on Pattern Analysis and Machine Intelligence, vol. 24, pp. 402-412, 2002.

[32] R. Cappelli, M. Ferrara, A. Franco and D. Maltoni, "Fingerprint verification competition 2006", Biometric Technology Today, vol.15, no.7-8, pp.7-9, August 2007.

[33] Arun A. Ross, Karthik Nandakumar, and Anil K. Jain. Handbook of Multibiometrics (International Series on Biometrics). Springer-Verlag New York, Inc., Secaucus, NJ, USA, 2006.

[34] S. Mazumdar and V. Dhulipala, "Biometric security using fingerprint recognition," 3, 2008

[35] H. Patel and P. Asrodia, "Fingerprint matching using two methods," Vol. 2, No. 3, pp. 857-860, 2012.

[36] S. Bana and D. Kaur, "Fingerprint recognition using image segmentation," International Journal Of Advanced Engineering Sciences And Technologies, Vol. 5, pp. 012-023, 2011.

[37] Azzoubi, Einas Almarghni, And Rosziati Bint Ibrahim. "An Enhancement Algorithm Using Gabor Filter For Fingerprint Recognition." Journal of Theoretical and Applied Information Technology 74.3 (2015).

[38] Kulshrestha, Megha, V. K. Banga, and Sanjeev Kumar. "Finger Print Recognition: Survey of Minutiae and Gabor Filtering Approach." International Journal of Computer Applications 50.4 (2012).

[39] J.venkatesh A Study and Analysis of Gabor Filter and KNearest Neighbor Approach on Minutia Matching for Fingerprint Recognition Indian Journal of Applied Research, Vol.III, Issue.IX September 2013

[40] M. Haghighat, S. Zonouz, M. Abdel-Mottaleb, "CloudID: Trustworthy cloud-based and cross-enterprise biometric identification," Expert Systems with Applications, vol. 42, no. 21, pp. 7905-7916, 2015.

[41] Kaggwa, F.; Ngubiri, J.; Tushabe, F., "Evaluation of multiple enrollment for fingerprint recognition," Computer \& Information Technology (GSCIT), 2014 Global Summit on , vol., no., pp.1,6, 1416 June 2014

[42] Kaggwa, F.; Ngubiri, J.; Tushabe, F. (2015, Mar.) Multiple enrollment based Fingerprint Recognition Systems: State of the Art Survey. IJCIT Volume 04-Issue 02 [Online]. Available: http://www.ijcit.com/archives/volume4/issue2/Paper0402 40.pdf

[43] Kaggwa, F., Ngubiri, J. and Tushabe, F., 2015, June. Improving recognition performance in multiple enrollment based fingerprint recognition systems. In Computer \& Information Technology (GSCIT), 2015 Global Summit on (pp. 1-5). IEEE.

[44] A. K. Jain, S. Prabhakar, L. Hong and S. Pankanti, "Filterbank-based fingerprint matching," in IEEE Transactions on Image Processing, vol. 9, no. 5, pp. 846859, May 2000. 